STRUCTURAL BIOLOGY COMMUNICATIONS

ISSN 2053-230X

Received 28 February 2017

Accepted 30 March 2017

Edited by I. Tanaka, Hokkaido University, Japan

Keywords: RNA repair; CPDase; RNA 2', 3'cyclic phosphodiesterase; Deinococcus radiodurans.

PDB reference: native drCPDase, 5h7e

Supporting information: this article has supporting information at journals.iucr.org/f

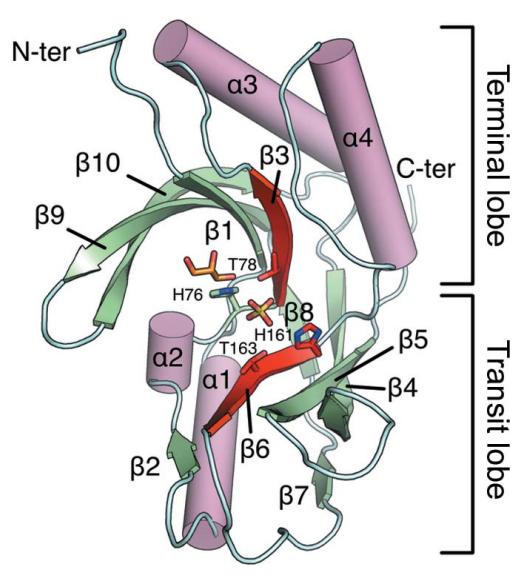

C 2017 International Union of Crystallography

\section{Crystal structure of the RNA $2^{\prime}, 3^{\prime}$-cyclic phosphodiesterase from Deinococcus radiodurans}

\author{
Wanchun Han, Jiahui Cheng, Congli Zhou, Yuejin Hua* and Ye Zhao*
}

Key Laboratory of Chinese Ministry of Agriculture for Nuclear-Agricultural Sciences, Institute of Nuclear-Agricultural Sciences, Zhejiang University, People's Republic of China. *Correspondence e-mail: yjhua@zju.edu.cn, yezhao@zju.edu.cn

$2^{\prime}, 3^{\prime}$-Cyclic phosphodiesterase (CPDase) homologues have been found in all domains of life and are involved in diverse RNA and nucleotide metabolisms. The CPDase from Deinococcus radiodurans was crystallized and the crystals diffracted to $1.6 \AA$ resolution, which is the highest resolution currently known for a CPDase structure. Structural comparisons revealed that the enzyme is in an open conformation in the absence of substrate. Nevertheless, the active site is well formed, and the representative motifs interact with sulfate ion, which suggests a conserved catalytic mechanism.

\section{Introduction}

Deinococcus radiodurans is well known for its extreme resistance to DNA-damaging treatments such as ionizing radiation (Ishino \& Narumi, 2015; Munteanu et al., 2015; Krisko \& Radman, 2013). It encodes a predicted $2^{\prime}-5^{\prime}$ RNA ligase (DR2339, LigT), which might be involved in RNA repair and metabolism (Makarova et al., 2001; Omelchenko et al., 2005). Comparative transcriptomics research suggests that the gene encoding this enzyme is highly upregulated in vivo following exposure to radiation (Chen et al., 2008; Liu et al., 2003). The LigT protein is conserved within the Deinococcus-Thermus phylum and is homologous to the Escherichia coli enzyme (Fig. 1). A recent proposal suggests that instead of performing a ligase reaction, E. coli LigT is actually a CPDase enzyme that converts an RNA 2',3'-cyclic phosphodiester into a $2^{\prime}-\mathrm{PO}_{4}$ terminus (Omelchenko et al., 2005).

CPDase (EC 6.5.1) is found in almost all organisms from bacteria to mammals and can exist as a 'standalone' protein in addition to forming part of tRNA ligase (Kato et al., 2003; Hofmann et al., 2000; Myllykoski et al., 2013; Remus et al., 2014; Mazumder et al., 2002). Based on domain arrangements and sequence alignments, CPDase is classified into the $2 \mathrm{H}$ phosphoesterase superfamily, which can be divided into four subfamilies: the archaeobacterial LigT-like, eukaryotic viral LigT-like, YjcG-like and mlr3352 groups (Mazumder et al., 2002). These subfamilies share two conserved histidines in a pair of $\mathrm{H} X \mathrm{~T} / \mathrm{S} X$ motifs (where $X$ is a hydrophobic residue), hence the name of this protein family. Superimpositions of these $2 \mathrm{H}$ phosphoesterase structures, which have been solved independently by nuclear magnetic resonance or X-ray crystallography in recent years, revealed that they share similar overall structures (Remus et al., 2014; Myllykoski et al., 2013, 2016; Sakamoto et al., 2005). The active site is located between two lobes formed by antiparallel $\beta$-strands with $\alpha$-helices wrapping around them. The two signature histidines serve as a general acid and a general base, respectively, which are critical for catalysis. 
Table 1

Macromolecule-production information.

\begin{tabular}{ll}
\hline Source organism & D. radiodurans (strain ATCC13939) \\
DNA source & Genomic DNA \\
Forward primer & 5'-GGAATTCCATATGAACAAGCCCGAAAAAAAGC \\
Reverse primer & 5'-AAGGATCCTCATGGGTGCTCCTGGGG \\
Cloning vector & pET-28a \\
Expression vector & pET-28a \\
Expression host & E. coli BL21 (DE3) \\
Complete amino-acid sequence & MGSSHHHHHSSGLVPRGSHMASMTGGQQMGRGS- \\
\cline { 2 - 2 } \multicolumn{1}{c}{ of the construct produced } & MNKPEKKPGSRAAQAGARPSRPAPRKQPGRE- \\
& SEHQPSTYRLFYALRVPADITAPLAEAQAKLR- \\
& GNWRAVRPDQMHVTLSYLPAVPERVEDLKRL- \\
& GTRLTQDLPPLHVNLRGTGYFNEGSPRVWFV- \\
& KTEAEGLTELAENLRAGIRELGIGTDDLAFKA- \\
& HITLARKKGPAPRLPPLIFDQSWTAPGLTLYR- \\
& SILRKTGPIYEVQSTFRFRGSASQTSAESAPT- \\
& AAPSAPQEHP \\
\hline
\end{tabular}

Table 2

Crystallization.

\begin{tabular}{ll}
\hline Method & Vapour diffusion, sitting drop \\
Plate type & 24 -well Linbro cell-culture plates \\
Temperature $(\mathrm{K})$ & 293 \\
Protein concentration $\left(\mathrm{mg} \mathrm{ml}^{-1}\right)$ & 25 \\
Buffer composition of protein & $20 \mathrm{~m} M$ Tris- $\mathrm{HCl} \mathrm{pH} \mathrm{7.5,250} \mathrm{mM} \mathrm{KCl,}$ \\
$\quad$ solution & $1 \mathrm{~m} M$ DTT, $0.5 \mathrm{~m} M$ EDTA \\
Composition of reservoir solution & $0.1 M$ citric acid pH 3.5, $2 M$ ammonium \\
& sulfate \\
Volume and ratio of drop & $1 \mu \mathrm{l}$ protein solution mixed with $1 \mu \mathrm{l}$ \\
& reservoir solution \\
Volume of reservoir $(\mu \mathrm{l})$ & 500 \\
\hline
\end{tabular}

In the present study, we determined the $1.6 \AA$ resolution crystal structure of CPDase from $D$. radiodurans (drCPDase), which shows an enlarged active site containing a sulfate ion that mimics the phosphate group of the substrate.

\section{Materials and methods}

\subsection{Macromolecule production}

Full-length drCPDase was amplified via PCR and cloned into pET-28a expression vector using NdeI and BamHI sites. Transformed E. coli BL21 (DE3) clones were grown at $37^{\circ} \mathrm{C}$ in LB medium containing $50 \mu \mathrm{g} \mathrm{ml}^{-1}$ kanamycin to an $\mathrm{OD}_{600}$ of between 0.6 and 0.8. Protein expression was induced at $37^{\circ} \mathrm{C}$ for $3 \mathrm{~h}$ by adding isopropyl $\beta$-D-1-thiogalactopyranoside (IPTG) to a final concentration of $0.3 \mathrm{~m} M$. The cell pellets were harvested and resuspended in buffer $A(20 \mathrm{~m} M$ Tris- $\mathrm{HCl}$ $\mathrm{pH} 7.8,9 \mathrm{~m} M$ imidazole, $500 \mathrm{~m} M \mathrm{NaCl}, 3 \mathrm{~m} M \beta$-mercaptoethanol) followed by sonication. After centrifugation at $35000 \mathrm{~g}$ for $30 \mathrm{~min}$, the supernatant was loaded onto an $\mathrm{Ni}-$ NTA column ( $5 \mathrm{ml}$; GE Healthcare Life Sciences) equilibrated with buffer $B$ [20 m $M$ Tris- $\mathrm{HCl} \mathrm{pH} 7.5,500 \mathrm{~m} M \mathrm{NaCl}$, $5 \%(w / v)$ glycerol, $3 \mathrm{~m} M \beta$-mercaptoethanol, $20 \mathrm{~m} M$ imidazole], washed with $40 \mathrm{~m} M$ imidazole and finally eluted with $300 \mathrm{~m} M$ imidazole. Fractions containing drCPDase were collected and were purified using a Superdex 75 10/30 column (GE Healthcare Life Sciences) with buffer $C$ (20 m $M$ Tris$\mathrm{HCl} \mathrm{pH} \mathrm{7.5,} 250 \mathrm{~m} M \mathrm{KCl}, 1 \mathrm{~m} M$ DTT, $0.5 \mathrm{~m} M$ EDTA). Macromolecule-production information is summarized in Table 1.
Table 3

Data collection and processing.

\begin{tabular}{ll}
\hline Diffraction source & BL17U, SSRF \\
Wavelength $(\AA)$ & 0.9792 \\
Temperature $(\mathrm{K})$ & 95 \\
Detector & ADSC Quantum 315r CCD \\
Crystal-to-detector distance $(\mathrm{mm})$ & 220 \\
Rotation range per image $\left({ }^{\circ}\right)$ & 0.5 \\
Total rotation range $\left({ }^{\circ}\right)$ & 90 \\
Exposure time per image $(\mathrm{s})$ & 1 \\
Space group & $H 32$ \\
$a, b, c(\AA)$ & $91.32,91.32,169.83$ \\
$\alpha, \beta, \gamma\left({ }^{\circ}\right)$ & $90,90,120$ \\
Mosaicity $\left({ }^{\circ}\right)$ & 0.172 \\
Resolution range $(\AA)$ & $30-1.600(1.640-1.600)$ \\
Total No. of reflections & 194852 \\
No. of unique reflections & 34987 \\
Completeness $(\%)$ & $96.7(97.3)$ \\
Multiplicity & $5.6(5.5)$ \\
$\langle I / \sigma(I)\rangle$ & $19.40(2.49)$ \\
$R_{\text {r.i.m. }}$ & $0.049(0.72)$ \\
Overall $B$ factor from Wilson plot $\left(\AA^{2}\right)$ & 23.30
\end{tabular}

Table 4

Structure refinement.

\begin{tabular}{ll}
\hline Resolution range $(\AA)$ & $30-1.600$ \\
Completeness $(\%)$ & 96.82 \\
No. of reflections, working set & $33239(2736)$ \\
No. of reflections, test set & $1738(149)$ \\
$R_{\text {work }}$ & 0.1747 \\
$R_{\text {free }}$ & 0.1963 \\
No. of non-H atoms & \\
$\quad$ Protein & 1405 \\
$\quad$ Glycerol & 12 \\
$\quad$ Sulfate & 25 \\
$\quad$ Waters & 118 \\
$\quad$ Total & 1560 \\
R.m.s. deviations & \\
$\quad$ Bonds $(\AA)$ & 0.015 \\
$\quad$ Angles $\left({ }^{\circ}\right)$ & 1.780 \\
Average $B$ factors $\left(\AA^{2}\right)$ & \\
$\quad$ Glycerol & 70.60 \\
$\quad$ Sulfate & 63.63 \\
Ligand & 65.89 \\
$\quad$ Waters & 39.17 \\
Ramachandran plot & \\
$\quad$ Most favoured $(\%)$ & 97.78 \\
Allowed $(\%)$ & 2.22 \\
\hline
\end{tabular}

\subsection{Crystallization}

An initial crystallization screen was performed using the sitting-drop vapour-diffusion method at $293 \mathrm{~K}$. drCPDase crystals were obtained using a reservoir consisting of $0.1 \mathrm{M}$ citric acid $\mathrm{pH}$ 3.5, $2 \mathrm{M}$ ammonium sulfate. Cryocooling was achieved by soaking the crystals in reservoir solution containing $30 \%$ glycerol for $3 \mathrm{~min}$ and flash-cooling them in liquid nitrogen. Crystallization information is summarized in Table 2.

\subsection{Data collection and processing}

Diffraction intensities were recorded on beamline BL17U at Shanghai Synchrotron Radiation Facility (SSRF), Shanghai, People's Republic of China and were integrated and scaled using the XDS suite (Kabsch, 2010). Data-collection and processing statistics are summarized in Table 3. 


\subsection{Structure solution and refinement}

The structure of drCPDase was determined by molecular replacement using the E. coli homologue (PDB entry 4qak; Remus et al., 2014) as a search model. The structure was refined using PHENIX interspersed with manual model building using Coot (Emsley et al., 2010; Adams et al., 2010). All structural figures were rendered in PyMOL (Schrödinger), and electrostatic surface potentials were calculated using APBS (Baker et al., 2001). The final drCPDase model consisted of residues 35-216, and all of the residues were in the most favourable and allowed regions of the Ramachandran plot. The coordinates and structure factors have been deposited in the Protein Data Bank with accession code 5h7e. The refinement statistics are listed in Table 4.

\section{Results and discussion}

drCPDase is a 234-amino-acid protein that shares $33 \%$ aminoacid identity with the $E$. coli homologue (Fig. 1). A sequence alignment shows that in addition to the two representative $2 \mathrm{H}$ phosphoesterase motifs, CPDases from the DeinococcusThermus phylum possess additional $\mathrm{N}$ - and $\mathrm{C}$-terminal regions outside the core domain (Fig. 1). To determine the crystal structure of drCPDase, the full-length protein was expressed and purified by metal-affinity and size-exclusion chromatography as described in $\$ 2$. Crystals were grown and diffracted X-rays to $1.6 \AA$ resolution and the structure was determined by molecular replacement. After refinement, the final model consisted of drCPDase residues 35-216 (the core domain). The N-terminal (amino acids 1-34) and C-terminal (amino acids 217-234) regions appear to be disordered in the crystal (Fig. 2a). The structure was validated by the appearance of a well formed active site containing a sulfate ion, which was present at $2 M$ in the crystallization solution, mimicking the phosphate group of the substrate (Fig. 2a).

The overall structure of the drCPDase core domain comprises two symmetrical lobes with four $\alpha$-helices and ten $\beta$-strands (Fig. $2 a$ ). The lobe containing the $\mathrm{N}$ - and C-terminal regions (the so-called terminal lobe; Hofmann et al., 2000) consists of four $\beta$-strands $(\beta 1, \beta 3, \beta 9$ and $\beta 10)$ and two $\alpha$-helices ( $\alpha 3$ and $\alpha 4$ ), and the opposite lobe (the so-called

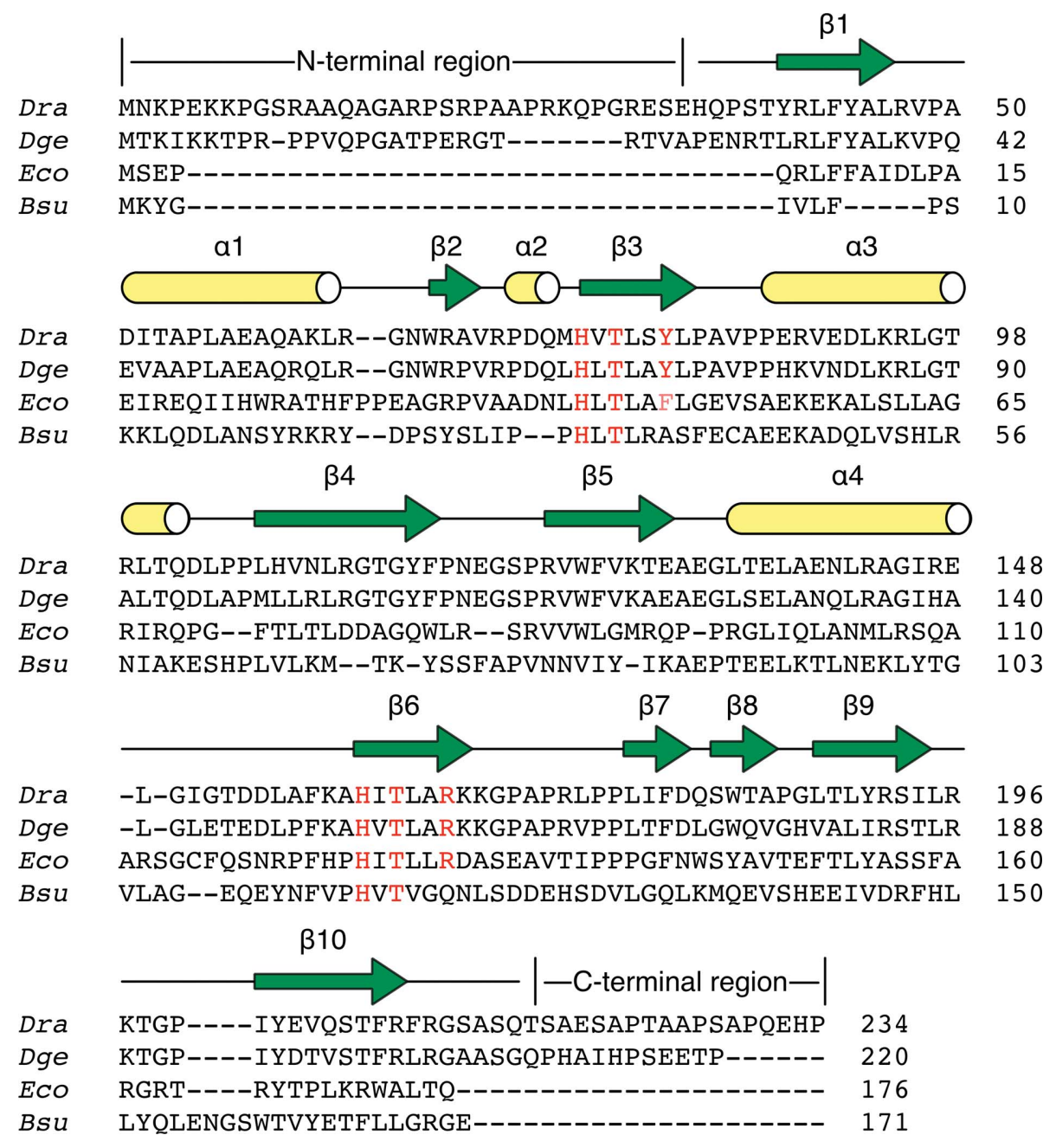

Figure 1

Sequence alignments of CPDase-family proteins from D. radiodurans (Dra), D. geothermalis (Dge), E. coli (Eco) and Bacillus subtilis (Bsu). The structural elements of drCPDase are shown in distinct colours. Conserved amino acids are highlighted in red. 
transit lobe) consists of six $\beta$-strands ( $\beta 2, \beta 4, \beta 5, \beta 6, \beta 7$ and $\beta 8$ ) and two $\alpha$-helices $(\alpha 1$ and $\alpha 2)$. Four of the antiparallel $\beta$-strands from each lobe $(\beta 1-\beta 3-\beta 9-\beta 10$ and $\beta 4-\beta 5-\beta 6-\beta 8)$

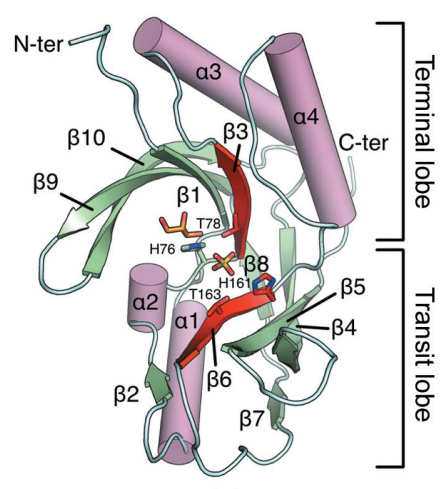

(a)

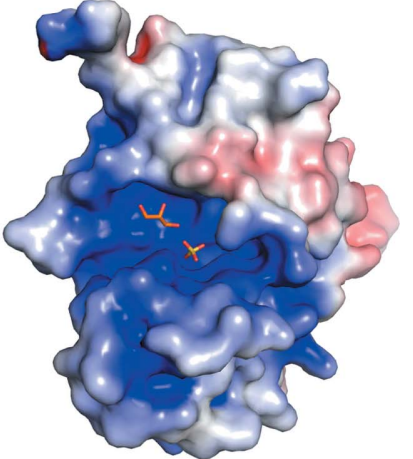

(b)

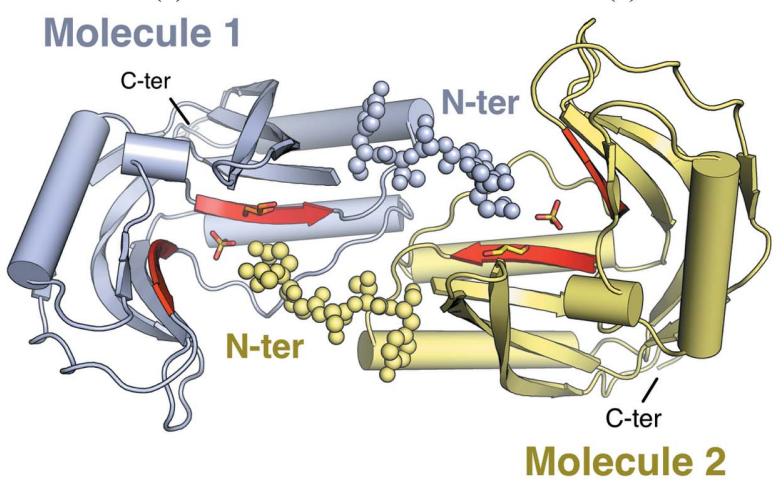

(c)

Figure 2

Structural features of drCPDase. (a) The overall structure of drCPDase is shown with green $\beta$-strands, pink $\alpha$-helices and cyan loop regions. The two $\beta$-strands bearing the signature motifs are highlighted in red. The Nand $\mathrm{C}$-termini of the polypeptide are indicated. The catalytic residues, sulfate and glycerol in the active site are depicted as stick models. (b) The distribution of the electrostatic surface of drCPDase. Blue and red represent the positive and negative charge potentials on a +8 and $-8 k \mathrm{ke}^{-1}$ scale, respectively. (c) The two symmetry-related drCPDase molecules in the H32 crystal are coloured grey and yellow. The $\mathrm{N}$-terminal region (residues 35-40) of each molecule is shown as spheres. constitute the solvent-accessible $\beta$-barrel architecture. The two conserved catalytic $\mathrm{H} X \mathrm{~T} / \mathrm{S} X$ motifs are located in the $\beta 3$ and $\beta 6$ strands (highlighted in red in Fig. $2 a$ ), which form a large, positively charged active-site cleft for substrate binding (Fig. 2b). In addition, a molecule of glycerol (used for cryoprotection) was observed to be hydrogen-bonded to the sulfate ion in the active site. Interestingly, the N-terminal disordered region before the drCPDase core domain points towards the active site of the symmetry-related protein molecule (Fig. 2c), suggesting that the $\mathrm{N}$-terminal region might play a role in protein function. In fact, the $\mathrm{N}$-terminal domain of CNPase, a member of the $2 \mathrm{H}$ phosphoesterase family, plays a regulatory role in substrate binding (Myllykoski et al., 2013).

We next compared the drCPDase structure with that of its E. coli homologue (ecCPDase), which has been recently solved in complex with 2'-AMP (Remus et al., 2014). The overall structure of drCPDase could be superimposed on that of ecCPDase with an r.m.s.d. of $1.213 \AA$ over $104 \mathrm{C}^{\alpha}$ atoms (Fig. 3a). Despite the differing numbers of $\beta$-strands, drCPDase shares a conserved active-site topology with ecCPDase (Fig. 3a). The central $\beta$-barrel superimposes well in the two structures. However, the flanking helices, especially $\alpha 1$ and $\alpha 3$, showed noticeable movements that create a wider substrate-entrance channel (Fig. 3a). Compared with ecCPDase, the active-site cavity of drCPDase is filled with water, glycerol and sulfate ion in the absence of $2^{\prime}$-AMP (Figs. $3 a$ and $3 b$ ). The sulfate ion occupies a similar position to the phosphate group of $2^{\prime}$-AMP in ecCPDase, which is coordinated by a glycerol molecule and conserved residues (Thr78, His161, Thr163 and Arg166) from the two signature motifs (Fig. 3b). Notably, all of the residues that are likely to be involved in the CPDase reaction can be superimposed in drCPDase and ecCPDase, which would suggest that CPDase has an open substrate-binding site in the catalytic domain. This active-site configuration is likely to be conserved among all CPDases and possibly also all $2 \mathrm{H}$ phosphoesterases. In the absence of $2^{\prime}$-AMP, the $\alpha$-helices in drCPDase show a

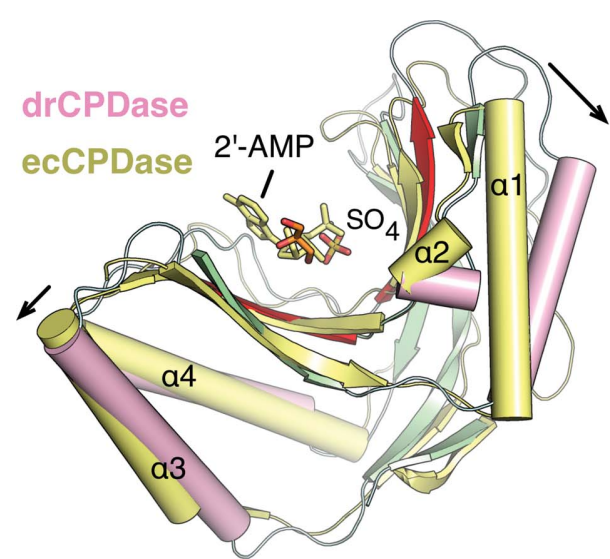

(a)

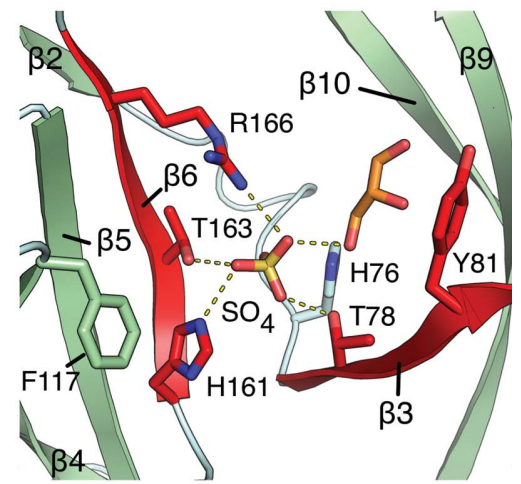

(b)

Figure 3

Structural basis for substrate recognition by drCPDase. (a) Structural comparison of drCPDase (coloured as in Fig. 2a) and ecCPDase (yellow). The relative domain movements are shown with arrowheads. (b) Close-up of the drCPDase active site between two $\beta$-strands (red). The conserved residues and the sulfate ion are shown as stick models. 
noticeable movement and result in a more open passage for substrate binding, indicating that these $\alpha$-helices are also involved in substrate recognition.

In summary, we have determined the crystal structure of the CPDase homologue from $D$. radiodurans at $1.6 \AA$ resolution. The structure delineates an open active site for substrate binding with a conserved catalytic mechanism. These results will aid in future biochemical analyses of CPDase as well as other members of the $2 \mathrm{H}$ phosphoesterase superfamily.

\section{Acknowledgements}

We would like to thank the staff at the Shanghai Synchrotron Radiation Facility (SSRF) in China for assistance in data collection.

\section{Funding information}

Funding for this research was provided by: National Basic Research Program of China (award No. 2015CB910600); Zhejiang Provincial Natural Science Foundation for Outstanding Young Scientists (award No. LR16C050002); National Natural Science Foundation of China (award No. 31670819, 31670065, 31500656, 31370102, 31210103904).

\section{References}

Adams, P. D. et al. (2010). Acta Cryst. D66, 213-221.

Baker, N. A., Sept, D., Joseph, S., Holst, M. J. \& McCammon, J. A. (2001). Proc. Natl Acad. Sci. USA, 98, 10037-10041.
Chen, H., Xu, G., Zhao, Y., Tian, B., Lu, H., Yu, X., Xu, Z., Ying, N., Hu, S. \& Hua, Y. (2008). PLoS One, 3, e1602.

Emsley, P., Lohkamp, B., Scott, W. G. \& Cowtan, K. (2010). Acta Cryst. D66, 486-501.

Hofmann, A., Zdanov, A., Genschik, P., Ruvinov, S., Filipowicz, W. \& Wlodawer, A. (2000). EMBO J. 19, 6207-6217.

Ishino, Y. \& Narumi, I. (2015). Curr. Opin. Microbiol. 25, 103-112.

Kabsch, W. (2010). Acta Cryst. D66, 125-132.

Kato, M., Shirouzu, M., Terada, T., Yamaguchi, H., Murayama, K., Sakai, H., Kuramitsu, S. \& Yokoyama, S. (2003). J. Mol. Biol. 329, 903-911.

Krisko, A. \& Radman, M. (2013). Cold Spring Harb. Perspect. Biol. 5, $\mathrm{a} 012765$

Liu, Y., Zhou, J., Omelchenko, M. V., Beliaev, A. S., Venkateswaran, A., Stair, J., Wu, L., Thompson, D. K., Xu, D., Rogozin, I. B., Gaidamakova, E. K., Zhai, M., Makarova, K. S., Koonin, E. V. \& Daly, M. J. (2003). Proc. Natl Acad. Sci. USA, 100, 4191-4196.

Makarova, K. S., Aravind, L., Wolf, Y. I., Tatusov, R. L., Minton, K. W., Koonin, E. V. \& Daly, M. J. (2001). Microbiol. Mol. Biol. Rev. 65, 44-79.

Mazumder, R., Iyer, L. M., Vasudevan, S. \& Aravind, L. (2002). Nucleic Acids Res. 30, 5229-5243.

Munteanu, A., Uivarosi, V. \& Andries, A. (2015). Extremophiles, 19, 707-719.

Myllykoski, M., Raasakka, A., Lehtimäki, M., Han, H., Kursula, I. \& Kursula, P. (2013). J. Mol. Biol. 425, 4307-4322.

Myllykoski, M., Seidel, L., Muruganandam, G., Raasakka, A., Torda, A. E. \& Kursula, P. (2016). Brain Res. 1641, 64-78.

Omelchenko, M. V., Wolf, Y. I., Gaidamakova, E. K., Matrosova, V. Y., Vasilenko, A., Zhai, M., Daly, M. J., Koonin, E. V. \& Makarova, K. S. (2005). BMC Evol. Biol. 5, 57.

Remus, B. S., Jacewicz, A. \& Shuman, S. (2014). RNA, 20, 1697-1705. Sakamoto, Y., Tanaka, N., Ichimiya, T., Kurihara, T. \& Nakamura, K. T. (2005). J. Mol. Biol. 346, 789-800. 